\title{
INSZENIERUNG
}

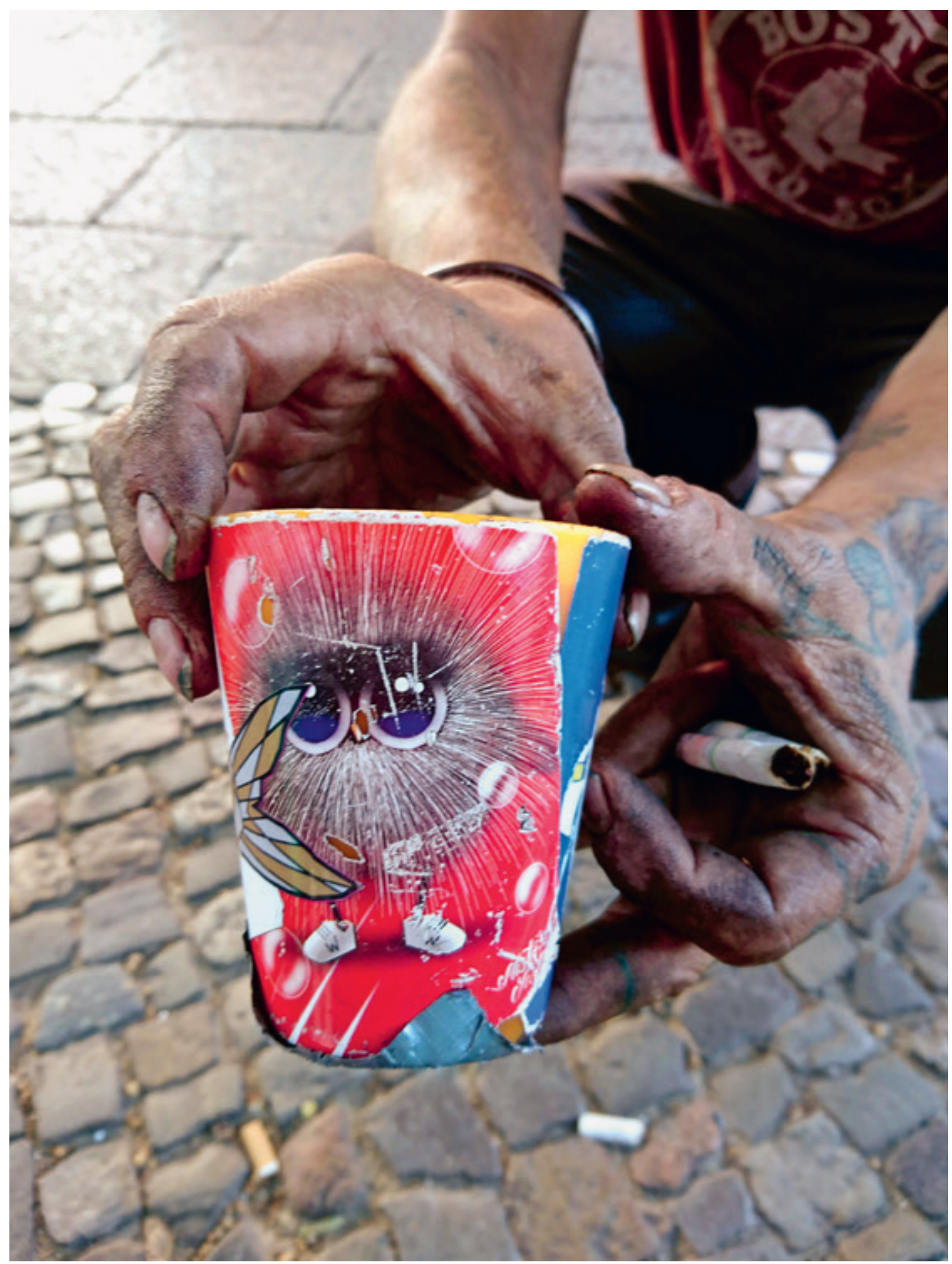




\section{JÜRGEN KRUSCHE}
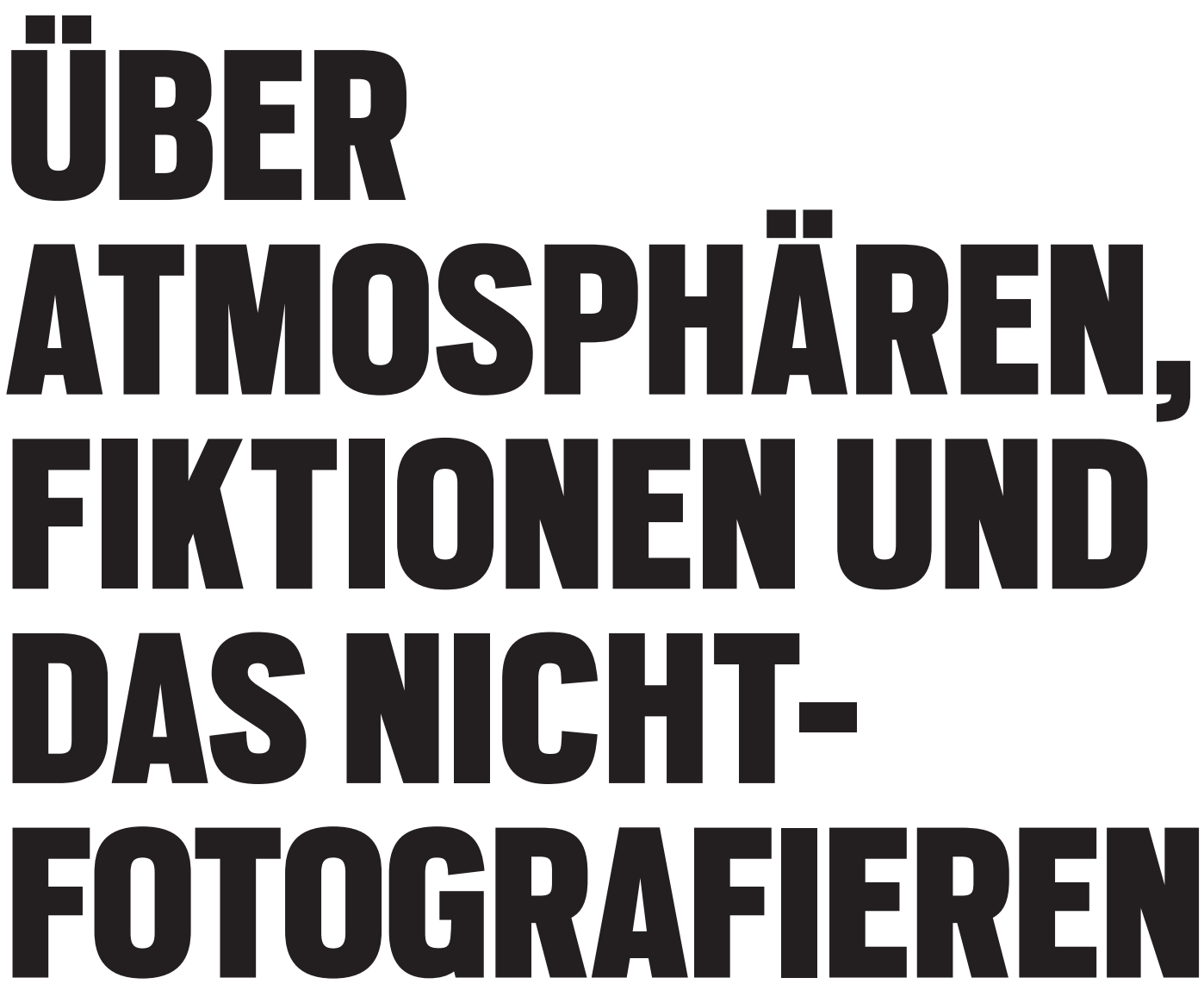
Die Transformation unserer Städte lässt sich an verschiedenen Indizien ablesen: an der baulichen Substanz, an der Gestaltung der öffentlichen Räume, an Statistiken zur Veränderung der Sozialstruktur von Quartieren oder an subjektiven Erfahrungen, wie sie in diesem Buch vorgestellt werden. Die Veränderung der Städte weltweit kann aber auch an deren charakteristischen Atmosphären festgestellt werden, zu denen vor allem die öffentlichen Räume beitragen. Viele Städte sind im Vergleich zu früher fußgängerfreundlicher geworden, Plätze wurden von Autos befreit, die Aufenthaltsqualität der Innenstädte und Fußgängerbereiche wurde verbessert, Grünanlagen und Parks wurden erstellt - mit allen Vor- und Nachteilen, die diese Neugestaltungen mit sich bringen.

\section{Atmosphären}

Aber auch andere Phänomene prägen städtische Atmosphären. Im Fokus dieser Bildarbeit stehen jene Atmosphären, die vor allem durch die Anwesenheit von Exkludierten (Bettelnde, Obdach- und Wohnungslose, PfandflaschensammlerInnen, Drogen- und Alkoholabhängige) und ihren Gegenständen, die sie oft bei sich führen, entstehen. Philosophisch betrachtet ist die Atmosphäre etwas, das „dazwischen“ liegt, zwischen der materiellen Umgebung und dem subjektiven Empfinden; sie ist weder rein objektiv noch rein subjektiv. ${ }^{1}$ In der 1 „Die Atmosphären sind so konzipiert weder gepflegten Innenstadt verändert ein am Boden kauern- etwas objektives, nämlich der Bettelnder mit einem Becher vor seinen Füßen die Atmosphäre, aber auch das Verhalten der Menschen, die diese „Figur“ am Boden wahrnehmen. Es entsteht eine Spannung oder Stimmung, die nicht objektivierbar, aber wahrnehmbar und durch die Anwesenheit des Bettelnden auch sichtbar ist. Es ist nicht allein der Mensch am Boden - das objektiv Gegebene -, sondern auch die Wahrnehmung der Vorbeigehenden - das Subjektive -, Eigenschaften, die die Dinge haben, und doch sind sie etwas Dinghaftes, zum Ding Gehöriges, insofern nämlich die Dinge durch ihre Eigenschaften als Ekstasen gedacht - die Sphären ihrer Anwesenheit artikulieren. Noch sind die Atmosphären etwas Subjektives, etwa Bestimmungen eines Seelenzustands." Böhme, Gernot (1995): Atmosphäre,

Frankfurt am Main, S. 33 
2 Siehe dazu: Hasse, Jürgen (2017): „Autopsien der Stadt. Fotografische Mikrologien“, in: Krusche, Jürgen: Die ambivalente Stadt, Berlin, S. 132-157 und Ders. (2012): Atmosphären der Stadt. Aufgespürte Räume, Berlin

welche erst im Zusammenspiel die Atmosphäre erzeugen. Die Fotografie, genauer gesagt eine Abfolge von Fotografien, scheint dafür geeignet zu sein, diese Atmosphären nicht nur zu „zeigen“, sondern sie bis zu einem gewissen Grad auch durch das subjektive Empfinden der Betrachtenden nachvollziehbar zu machen ${ }^{2}$ - ein Versuch, der hier unternommen wurde.

Im Wesentlichen wurden für diese Arbeit zwei Methoden/Strategien gewählt: die inszenierte Fotografie für die Serie „Der Stellvertreter" und die klassische Dokumentation - zum Teil auch mit dem Smartphone - für die Alltagsgegenstände wie in den Serien „Bettel-Becher“ oder „Orte“. Beide Serien werden, ergänzt durch Skizzen und weiteres Arbeitsmaterial, für diesen Beitrag zu einem Bild-Essay zusammengefügt.

\section{Der Stellvertreter}

Die Arbeit „Der Stellvertreter“ tangiert verschiedene Diskurse und führt sie in einem Bild/einer Serie zusammen: Kunst und Ethnographie, Fiktion und Realität, Inszenierung, Nutzung des öffentlichen Raums, Armut, Tourismus und Konsum, Inklusion und Exklusion.

Die Fotografien zeigen öffentliche, belebte wie unbelebte urbane Räume mit gewöhnlichen Nutzungen. Doch dazwischen gibt es einen Störfaktor: Da sitzt, liegt, steht ein Mensch, der nicht diesen gewöhnlichen Nutzungen nachgeht. Er sitzt anteilslos, in sich versunken am Boden, wartet oder bettelt; er stört die Szenerie, die von Geschäftigkeit geprägt ist, er „infiziert“ das Bild und lässt eine spezifische Atmosphäre entstehen.

Beim Betrachten der Serie wird klar, dass es sich hier immer um die gleiche Person handelt, dass es keine gewöhnliche Dokumentarfotografie ist; es ist nicht einfach ein Abbild. Die sitzende Figur ist absichtlich dort platziert worden, die Situation 
ist inszeniert. Hier wird nicht „wirklich“ gebettelt, sondern nur so getan als ob. Die Haltungen, Gesten werden nachgestellt. Die Orte, an denen diese stattfinden, sind jedoch „authentisch“. Es sind Orte, an denen sonst tatsächlich Menschen sitzen, die betteln, Menschen, die nicht am Konsum teilhaben, die nicht in der üblichen Art und Weise in diesen öffentlichen Räumen agieren, sondern einfach da sind: mit einem Pappbecher, einem Rucksack oder einer Bierflasche - oder mit leeren Händen.

Die „Anteilslosen“ (Rancière) bilden das Zentrum dieser Arbeit, so, wie sie sich im öffentlichen Raum aufhalten: mittendrin und abseits zugleich, anwesend, aber dennoch unsichtbar. Von den PassantInnen werden sie kaum beachtet; das urbane Leben zieht an ihnen vorüber. Sie scheinen nicht Teil dieser Gesellschaft zu sein, nicht integriert, sondern exkludiert.

\section{Ethnofiktion}

Neben dieser inhaltlichen Ebene wird auch die Frage nach dem Verhältnis von Fiktion und Wirklichkeit gestellt. Warum sitzt da ein „Stellvertreter“ und nicht ein „realer“ Obdachloser oder eine „echte“ Trinkerin, wie wir sie doch alle täglich sehen? Weil es einen Unterschied macht, ob wir diese Menschen sehen oder fotografieren. Sie sollen nicht ausgestellt werden, eingefroren in einem Bild, ohne eigene Stimme. Der „Stellvertreter“ hält das aus, er ist Schauspieler und darf gezeigt werden. Er stellt die Situation nach. Ort und Haltung sind „echt“, die Person ist es nicht. Dennoch wird das Wesentliche gesagt. Das Bild zeigt die Atmosphäre, wie sie sich durch diese spezielle Konstellation aufbaut; es zeigt die Störung, das (un-)gewöhnliche Nebeneinander von Armut und Konsum, von Rand und Mitte, von Sicht- und Unsichtbarkeit, von In- und Exklusion.

Auslöser für diese Art des Arbeitens war die Verweigerung, Exkludierte zu fotografieren. Es sollte weder eine Dokumentation 
noch eine Reportage werden; auch sollten für einmal nicht die Person und ihre Biografie im Mittelpunkt stehen, sondern der Ort und seine Atmosphäre. Als Ausweg aus diesem Dilemma schien sich die Fiktion anzubieten, so wie sie Marc Augé in sei-

3 Augé, Marc (2012): Tagebuch eines Obdachlosen. Ethnofiktion, München

4 Edwards, Steve (2012): Martha Rosler. The Bowery in two inadequate descriptive systems, London, S. 12 5 Zitiert nach Szepanski, Birgit (2016): Erzählte Stadt. Der urbane Raum bei Janet Cardiff und Jeff Wall, Bielefeld, S. 249

nem Tagebuch eines Obdachlosen eingesetzt hat. ${ }^{3}$

Weitere Inspirationen kamen aus der Kunst, etwa von Martha Roslers Foto-Text-Arbeit The Bowery in two inadaquate descriptive systems (1974-75), die verdeutlicht, dass auch ohne „Dokumentar-Pornographie“ (documentary ,pormography $)^{4}$ etwas "gezeigt" werden kann. Auch die Arbeiten von Jeff Wall waren sehr anregend und führten dazu, dass der Versuch unternommen wurde, mit einem Schauspieler zu arbeiten. Seine Herangehensweise war eine wichtige Inspirationsquelle, die mein Vorhaben bestätigte. In einem Interview über seine Arbeit sagte er: „Ich beginne beim Nicht-Fotografieren." (I begin by not photographing.) ${ }^{5}$

So stand auch bei dieser Serie das „Nicht-Fotografieren“ am Anfang. Auf der Basis von ausgedehnten Feldforschungen entstanden zunächst Skizzen, die typische Haltungen von Exkludierten und die dazu passenden Orte festhielten. Basierend auf diesen Skizzen wurden später mit dem Schauspieler an exakt diesen Orten die Positionen und Haltungen der Exkludierten reinszeniert. Der Schauspieler macht nur einen kleinen Teil des Bilds aus. Nur er ist inszeniert, der gesamte Rest - der Ort und die vorübergehenden PassantInnen - ist „echt“. Entstanden ist eine Mischung aus inszenierter Fotografie und Street Photography.

Neben diesem Versuch, die Atmosphäre einzufangen, die durch die Präsenz einer Person entsteht, wurden auch Dinge, die in diesem Zusammenhang immer wieder Verwendung finden - etwa Becher, mit denen gebettelt wird -, im Sinne der 
Material Culture Studies dokumentiert. Aber auch Matratzen und andere Unterlagen, Schlaf- und Rucksäcke, Einkaufswagen und Plastiktüten und die dazugehörenden spezifischen Orte gehören in diesen Kontext.

Der nachfolgende Bildbeitrag versucht sowohl den Prozess des Forschens wie auch den des Entstehens dieser Bildarbeit aufzuzeigen. $\mathrm{Zu}$ sehen sind Ergebnisse, aber auch der Weg dorthin, auch Umwege und Korrektureingriffe. Der Beitrag versteht sich nicht allein als Fotografie, sondern als visualisierte Erkenntnisse im Sinne der künstlerischen Forschung im Medium der Fotografie. 
